\title{
Angioarchitecture of arteriovenous fistulas at the craniocervical junction: a multicenter cohort study of 54 patients
}

\author{
Masafumi Hiramatsu, MD, ${ }^{1}$ Kenji Sugiu, MD, ${ }^{1}$ Tomoya Ishiguro, MD, ${ }^{2}$ Hiro Kiyosue, MD, ${ }^{3}$ \\ Kenichi Sato, MD, ${ }^{4}$ Keisuke Takai, MD, ${ }^{5}$ Yasunari Niimi, MD, ${ }^{6}$ and Yuji Matsumaru, MD ${ }^{7}$ \\ 1Department of Neurological Surgery, Okayama University Graduate School of Medicine, Dentistry and Pharmaceutical \\ Sciences, Okayama; '2Department of Neuro-Intervention, Osaka City General Hospital, Osaka; ${ }^{3}$ Department of Radiology, Oita \\ University Faculty of Medicine, Oita; ${ }^{4}$ Department of Neuroendovascular Therapy, Kohnan Hospital, Sendai; ${ }^{5}$ Department of \\ Neurosurgery, Tokyo Metropolitan Neurological Hospital; ${ }^{6}$ Department of Neuroendovascular Therapy, St. Luke's International \\ Hospital; and ${ }^{7}$ Department of Endovascular Neurosurgery, Toranomon Hospital, Tokyo, Japan
}

OBJECTIVE The aim of this retrospective multicenter cohort study was to assess the details of the angioarchitecture of arteriovenous fistulas (AVFs) at the craniocervical junction (CCJ) and to determine the associations between the angiographic characteristics and the clinical presentations and outcomes.

METHODS The authors analyzed angiographic and clinical data for patients with CCJ AVFs from 20 participating centers that are members of the Japanese Society for Neuroendovascular Therapy (JSNET). Angiographic findings (feeding artery, location of AV shunt, draining vein) and patient data (age, sex, presentation, treatment modality, outcome) were tabulated and stratified based on the angiographic types of the lesions, as diagnosed by a member of the CCJ AVF study group, which consisted of a panel of 6 neurointerventionalists and 1 spine neurosurgeon.

RESULTS The study included 54 patients (median age 65 years, interquartile range 61-75 years) with a total of 59 lesions. Five angiographic types were found among the 59 lesions: Type 1, dural AVF (22 [37\%] of 59); Type 2, radicular AVF (17 [29\%] of 59); Type 3, epidural AVF (EDAVF) with pial feeders (8 [14\%] of 59); Type 4, EDAVF (6 [10\%] of 59); and Type 5, perimedullary AVF (6 [10\%] of 59). In almost all lesions (98\%), AV shunts were fed by radiculomeningeal arteries from the vertebral artery that drained into intradural or epidural veins through AV shunts on the dura mater, on the spinal nerves, in the epidural space, or on the spinal cord. In more than half of the lesions (63\%), the AV shunts were also fed by a spinal pial artery from the anterior spinal artery (ASA) and/or the lateral spinal artery. The data also showed that the angiographic characteristics associated with hemorrhagic presentations-the most common presentation of the lesions (73\%) - were the inclusion of the ASA as a feeder, the presence of aneurysmal dilatation on the feeder, and CCJ AVF Type 2 (radicular AVF). Treatment outcomes differed among the angiographic types of the lesions.

CONCLUSIONS Craniocervical junction AVFs commonly present with hemorrhage and are frequently fed by both radiculomeningeal and spinal pial arteries. The AV shunt develops along the C-1 or C-2 nerve roots and can be located on the spinal cord, on the spinal nerves, and/or on the inner or outer surface of the dura mater.

https://thejns.org/doi/abs/10.3171/2017.3.JNS163048

KEY WORDS craniocervical junction; arteriovenous fistula; radicular; pial feeder; aneurysmal dilatation; subarachnoid hemorrhage; vascular disorders

ABBREVIATIONS $A S A=$ anterior spinal artery; $A V=$ arteriovenous; $A V F=A V$ fistula; $A V M=$ arteriovenous malformation; $C C J=$ craniocervical junction; $D A V F=$ dural $A V F ;$ $\mathrm{DSA}=$ digital subtraction angiography; ECA = external carotid artery; EDAVF = epidural AVF; IQR = interquartile range; JSNET = Japanese Society for Neuroendovascular Therapy; LSA = lateral spinal artery; MIP = maximum intensity projection; $\mathrm{mRS}=$ modified Rankin Scale; PAVF = perimedullary AVF; RAVF = radicular AVF; RR = relative risk; $\mathrm{SAH}=$ subarachnoid hemorrhage; $\mathrm{VA}=$ vertebral artery.

SUBMITTED December 3, 2016. ACCEPTED March 23, 2017.

INCLUDE WHEN CITING Published online September 1, 2017; DOI: 10.3171/2017.3.JNS163048. 
$\mathrm{A}$ RTERIOVENOUS fistulas (AVFs) at the craniocervical junction $(\mathrm{CCJ})$ are rare vascular malformations found in $1 \%-2 \%$ of patients with intracranial or spinal AVFs. ${ }^{3,9,12}$ They are associated with acute neurological deficits related to hemorrhagic presentations (subarachnoid hemorrhage [SAH] and intramedullary hemorrhage) or slowly progressive myelopathy due to venous congestion, ${ }^{2,15}$ in contrast to spinal thoracolumbar AVFs that commonly present with venous congestion. ${ }^{7,8}$

Controversy exists regarding the diagnosis of $\mathrm{CCJ}$ AVFs given the complexity of their angioarchitecture. They can have intradural, dural, or epidural AV shunts that are fed by the spinal or dural artery and can be located on the spinal cord or dura mater. Prior reports have been based on a small number of patients treated by a single surgeon or at a single institution. The aim of the present retrospective multicenter cohort study was to assess the angioarchitecture of CCJ AVFs and to determine the associations between the angiographic characteristics and the clinical presentations and outcomes.

\section{Methods \\ Patient Population}

The study protocol was approved by the institutional review board at Toranomon Hospital. Because this was a retrospective noninvasive study, written informed consent was not obtained from patients. Participating centers were selected from among members of the Japanese Society for Neuroendovascular Therapy (JSNET) based on a recognized reputation in the treatment of vascular disorders and an expressed interest in study participation. A total of 81 patients with CCJ AVFs were treated at 20 centers in Japan between 2000 and 2015. Of these 81 patients, 54 patients with a total of 59 lesions met the inclusion criteria and were evaluated in this study. Patients were required to have CCJ AVFs defined as intradural, dural, or epidural AV shunt lesions without an intervening nidus located at the C-1 or C-2 vertebral levels. Intracranial AVFs such as marginal sinus AVFs or anterior condylar confluent AVFs or spinal AVFs at the C-3 and lower levels of the spine were excluded from our analysis. Intramedullary AV shunts were also excluded. Patients with angiographic findings without sufficient temporal and spatial resolution were also excluded.

\section{Data Collection}

Standardized forms were used to collect the clinical and radiological data, which included baseline patient characteristics (age, sex, presentation), treatment modality, outcome (modified Rankin Scale [mRS] scores at the onset of and 1 month after treatment), as well as angiography and MRI files in DICOM (Digital Imaging and Communications in Medicine) data format. Symptoms, when present, included SAH, intramedullary hemorrhage, venous congestion, and others.

\section{Angiographic Evaluation}

All collected angiographic data were reviewed using free DICOM viewer software (OsiriX Lite). Each patient was diagnosed by members of the CCJ AVF study group
(K. Sugiu, T.I., H.K., K. Sato, K.T., Y.N., and Y.M.), a panel consisting of 6 neurosurgeons and 1 neuroradiologistneurointerventionalists certified by the JSNET and a spine neurosurgeon certified by the Neurospinal Society of Japan. The CCJ AVFs were diagnostically defined according to the feeding arteries, presumed location of the AV shunts, and draining veins: dural AVF (DAVF), fed by meningeal arteries that drain into intradural veins through AV shunts located on the dura mater; radicular AVF (RAVF), fed by radicular and/or meningeal arteries that drain into radicular veins through AV shunts located on the spinal nerve roots; epidural AVF (EDAVF), fed by radicular and/or meningeal arteries that drain into epidural veins through AV shunts located outside the dura mater; perimedullary AVF (PAVF), fed by spinal pial arteries that drain into intradural veins through AV shunts located on the surface of the spinal cord. Locations of the AV shunts were presumed to be on the dura mater, on the spinal cord, or on the spinal nerves, based on the change in caliber between the feeding arteries and the draining veins.

\section{Statistical Analyses}

All calculations were performed using JMP 10 software (SAS Institute Inc.). Clinical and radiological data including baseline patient characteristics (age, sex, presentation), angiographic findings (spinal levels, laterality, feeding arteries, aneurysmal dilatation on the feeding artery, presence of a varix, and venous drainage of the AVF), treatment modality (surgery, endovascular treatment), and outcome (mRS scores) were summarized using descriptive statistics. Baseline patient characteristics, angiographic findings, and AVF type were assessed for association with hemorrhagic presentations based on univariate analyses. Cross-tabulations were generated for each variable, and the Fisher exact test was used. Results were presented as relative risk with $95 \%$ confidence intervals. Outcomes were assessed using the Wilcoxon signed-rank test. Statistical analyses were 2 -sided, with a $\mathrm{p}<0.05$ considered statistically significant.

\section{Results}

\section{Angioarchitecture}

Five angiographic types were found among the 59 lesions (Figs. 1-5): Type 1, DAVF (22 [37\%] of 59); Type 2, RAVF (17 [29\%] of 59); Type 3, EDAVF with pial feeders (8 [14\%] of 59); Type 4, EDAVF (6 [10\%] of 59); and Type 5, PAVF (6 [10\%] of 59).

As shown in Table 1, 42 (71\%) of 59 lesions were located at the C-1 level, and there was no laterality. Both DAVF (Type 1) and RAVF (Type 2) commonly developed at the C-1 level (100\% and 76\%, respectively), whereas EDAVF with or without pial feeders (Types 3 and 4) mainly developed at the C-2 level (75\% and $67 \%$, respectively). The arterial feeder of the lesions was the radiculomeningeal artery in 58 lesions (98\%), the anterior spinal artery (ASA) in 30 lesions (51\%), the lateral spinal artery (LSA) in 16 lesions $(27 \%)$, the ASA and/or LSA in 37 lesions $(63 \%)$, and the external carotid artery (ECA) in 7 lesions $(12 \%)$. The RAVFs, EDAVFs with pial feeders, and PAVFs were commonly fed by the pial feeder supplied from the ASA 


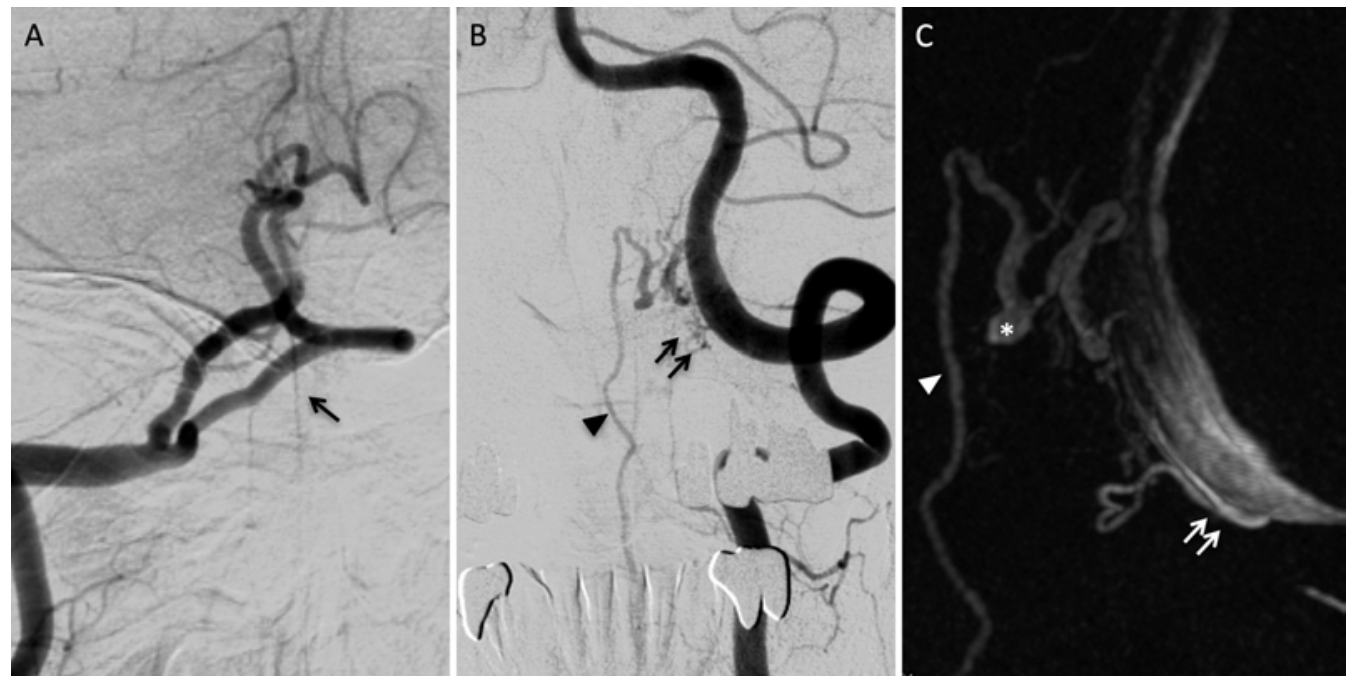

FIG. 1. Case 1. A CCJ DAVF (Type 1) presented with subarachnoid hemorrhage. Right vertebral artery (VA; A) and left VA (B) angiograms, anteroposterior views, and superselective angiogram $(\mathbf{C})$, oblique view, of the left $\mathrm{C}-1$ radiculomeningeal artery showing a DAVF. The AVF is fed by the left C-1 radiculomeningeal artery (double arrows in B and C) that drains into an intradural vein (arrowheads in B and C) with a varix (white asterisk in C). Note that the ASA (single arrow in A) from the contralateral right VA is not involved in the AVF. The AV shunt was presumed to be on the inner surface of the dura mater.

$(100 \%, 100 \%$, and $83 \%$, respectively) or the LSA (29\%, $38 \%$, and $33 \%$, respectively). Aneurysmal dilatation of the feeder was present on the pial artery (vasa corona) supplied from the ASA in 19 lesions $(32 \%)$.

Venous drainage of the lesions occurred through intradural drainage in 48 lesions $(81 \%), 43(73 \%)$ of which showed ascending drainage. Twenty-two lesions (37\%) had epidural drainage. Varices were present on the venous drainage in 10 lesions (17\%).

There were disagreements among the panel members regarding the diagnosis of angiographic Types 2 (RAVF) and 3 (EDAVF with pial feeders) based on the location of the AV shunts because of their complex angioarchitecture. In Type 2, the AVF was fed by the radicular or radiculomeningeal artery that drained into the intradural vein and by the pial branch of the ASA that drained into the same intradural vein (Fig. 2). Disagreement was resolved with subsequent consensus. We referred to the angiographic Type 2 as RAVF because it was interpreted as being fed by the radiculomeningeal artery that drains into the intradural vein through an AV shunt on the spinal nerve, where the pial branch of the ASA joins the same radicular artery and drains in a retrograde direction into the same intradural vein (Figs. 6A and B). Alternatively, the lesion may constitute a DAVF with pial feeders, which is fed by the radiculomeningeal artery that drains into the intradural vein through an AV shunt on the dura mater, where the pial branch of the ASA joins the same radiculomeningeal artery and drains into the same intradural vein (Figs. 6C and D). Another possibility was the presence of concurrent DAVFs and PAVFs.

In Type 3, the AVF was fed by the radiculomeningeal artery that drained into an epidural vein, and by the pial branch of the ASA that drained into the same epidural vein (Fig. 3). We referred to angiographic Type 3 as EDAVF with pial feeders because it was interpreted as being fed by the radiculomeningeal artery that drains into the epidural vein through an AV shunt outside the dura mater, where the branch of the ASA joins the same radiculomeningeal artery and drains into the same epidural vein.

\section{Clinical Characteristics}

As shown in Table 2, the median age at presentation was 65 years (interquartile range [IQR] 61-75 years), and there was a male predominance (76\%). The most common symptom was SAH (63\%), followed by venous congestion (12\%), intramedullary hemorrhage (10\%), and other symptoms (5\%). Six lesions (10\%) were asymptomatic. Hemorrhagic presentations (SAH and intramedullary hemorrhage) were more frequent in RAVF (94\%) and EDAVF with pial feeders $(87 \%)$, which had specific types of angioarchitecture, as described above.

\section{Treatment Modality and Outcome}

Of the 59 lesions, $28(47 \%)$ were treated with direct surgery only and $8(14 \%)$ were managed conservatively (Table 3). Fifteen lesions (25\%) were treated with endovascular embolization only. Eight lesions (14\%) were treated with both direct surgery and endovascular embolization. Glue was used as the embolic material for 17 lesions, and coils were used for 8 lesions. In patients with DAVF, RAVF, and EDAVF, direct surgery with or without interventional radiology was performed more frequently than endovascular embolization $(77 \%, 64 \%$, and $67 \%$, respectively). In contrast, in patients with EDAVF with pial feeders and in patients with PAVF, endovascular treatment was performed more often than direct surgery with or without embolization (50\% and 67\%, respectively).

Overall, mRS scores improved after treatment (median 3 [IQR 1-4] preoperatively vs 1 [IQR $0-3]$ postoperatively, $\mathrm{p}=0.0053$ ). In patients with DAVF, the mRS scores improved significantly (median 3 [IQR $1-3]$ preoperatively 

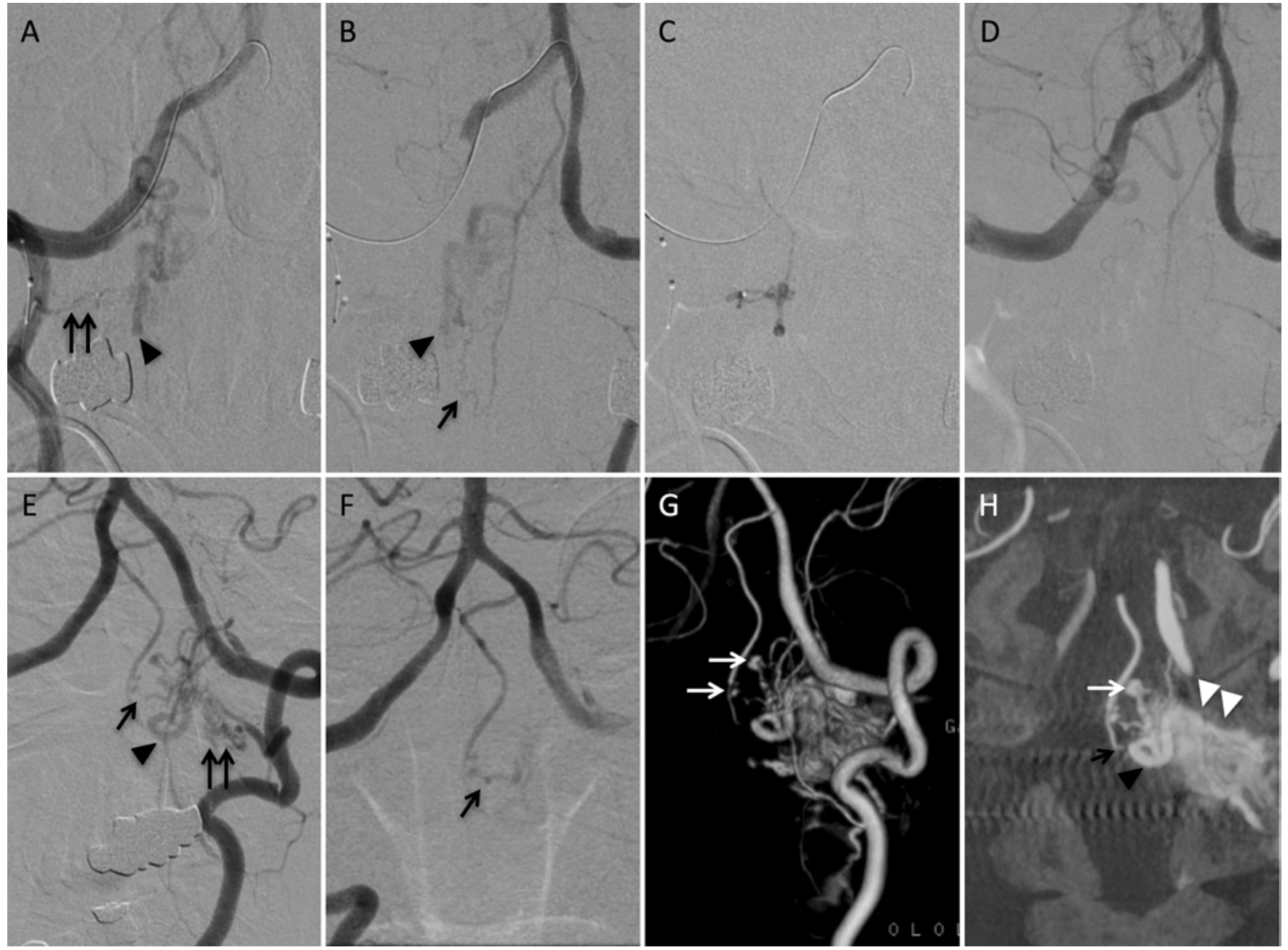

FIG. 2. Two cases of CCJ RAVF (Type 2) presented with subarachnoid hemorrhage. Case 2. Right VA (A) and left VA (B) angiograms, anteroposterior views, showing an RAVF. The AVF is independently fed by spinal pial arteries from the ASA (single arrow in B) and the right C-2 radiculomeningeal artery (double arrows in A). Both feeders drain into the same intradural vein (arrowheads in $\mathrm{A}$ and $\mathrm{B}$ ). Note that after endovascular embolization of the right $\mathrm{C}-2$ radiculomeningeal artery using glue (C), complete occlusion of the AVF was achieved (D), although spinal pial arteries from the ASA were left intact. The AV shunt was presumed to be located on the left C-2 nerve root. Case 3. Left VA angiogram (E), anteroposterior view, 3D digital subtraction angiography (DSA) image $(\mathbf{G})$, slab maximum intensity projection (MIP) image $(\mathbf{H})$, and right VA angiogram (F), anteroposterior view, showing an RAVF. The AVF is independently fed by spinal pial arteries from the ASA (single arrows in E and F) and the left C-2 radiculomeningeal artery (double arrows in $\mathrm{E}$ ). Both feeders drain into the same intradural vein (single black arrowheads in $\mathrm{E}$ and $\mathrm{H}$ ) and epidural venous plexus (double white arrowheads in $\mathrm{H}$ ). There are aneurysmal dilatations (single white arrows in $\mathrm{G}$ and $\mathrm{H}$ ) on the spinal arteries from the ASA (single black arrow in H). The AV shunt was presumed to be located on the right C-2 nerve root.

vs 2 [IQR 0.5-3] postoperatively, $\mathrm{p}=0.0389$ ). In patients with other angiographic types, the mRS scores did not significantly improve.

\section{Risk Factors Associated With Hemorrhagic Presentations}

As shown in Table 4, inclusion of the ASA as a feeder (relative risk [RR] 1.40, 95\% CI 1.02-1.92, $\mathrm{p}=0.04$ ), the presence of aneurysmal dilatation on the feeder (RR 1.64; 95\% CI 1.28-2.10, $\mathrm{p}=0.0012$ ), exclusion of the ECA as a feeder (RR 0.18, 95\% CI 0.03-1.09, $\mathrm{p}=0.001$ ), and the CCJ AVF Type 2, RAVF (RR 1.73, 95\% CI 1.16-2.57, p $=0.0106$ ), had a significant association with hemorrhagic presentations in univariate analyses. In contrast, intradural drainage (RR 0.87, 95\% CI 0.62-1.21, p = 0.71), ascending intradural drainage (RR 0.93, 95\% CI 0.67-1.29, p = 0.69), and the presence of a varix (RR $1.30,95 \%$ CI $0.98-1.71, p$ $=0.26$ ) did not.

\section{Discussion}

We provide details on the angioarchitecture of CCJ AVFs based on a multicenter cohort study of 59 lesions in 54 patients. We found 5 angiographic types of CCJ AVFs based on the feeding arteries, the presumed location of the AV shunts, and the draining veins: Type 1, DAVF; Type 2, RAVF; Type 3, EDAVF with pial feeders; Type 4, EDAVF; Type 5, PAVF (Figs. 1-5). In almost all lesions (98\%), the AV shunts were fed by radiculo- 

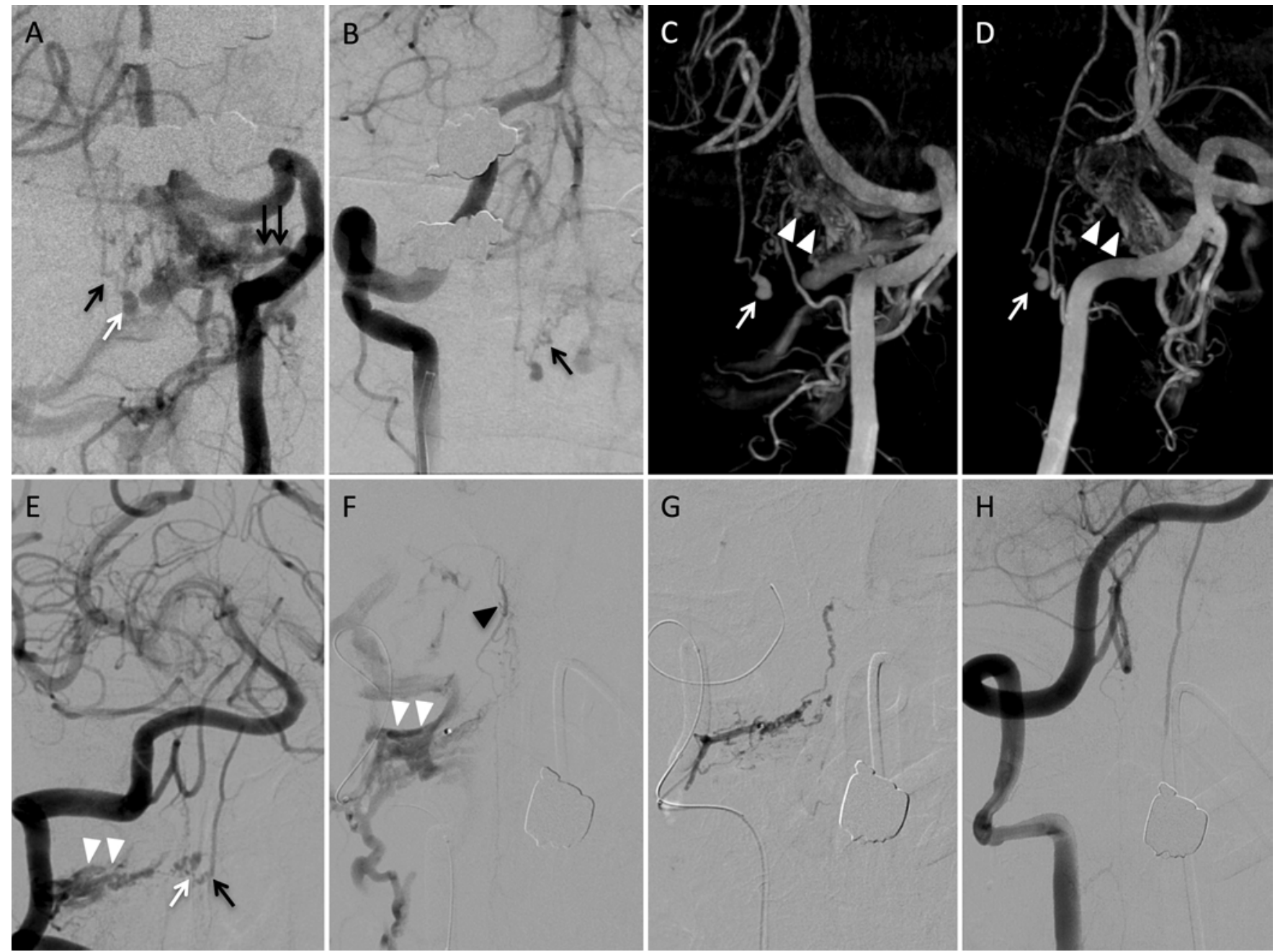

FIG. 3. Two cases of CCJ EDAVF with pial feeders (Type 3) presented with subarachnoid hemorrhage (Case 4) and intramedullary hemorrhage (Case 5). Case 4. Left VA angiogram (A), anteroposterior view, MIP images (C and D), and right VA angiogram (B), anteroposterior view, showing an EDAVF with pial feeders. The AVF is fed independently by spinal arteries from the ASA (single black arrows in $\mathrm{A}$ and $\mathrm{B}$ ) and the left $\mathrm{C}-2$ radiculomeningeal artery (double black arrows in A). Both feeders drain into the epidural venous plexus (double white arrowheads in $C$ and $D$ ). There is aneurysmal dilatation (single white arrows in $A, C$, and $D$ ) on the spinal pial arteries from the ASA. The AV shunt was presumed to be located in the epidural space. Case 5. Right VA angiogram $(E)$, anteroposterior view, and superselective angiogram of the right $C-2$ radiculomeningeal artery $(F)$ showing an EDAVF with pial feeders. The AVF is fed independently by spinal pial arteries from the ASA (single black arrow in E) and the right C-2 radiculomeningeal artery. Both feeders drain into the epidural venous plexus (double white arrowheads in $\mathrm{E}$ and $\mathrm{F}$ ) and intradural vein (black arrowhead in F). There is an aneurysmal dilatation (single white arrow in E) on the spinal pial arteries. Note that after endovascular embolization of the right C-2 radiculomeningeal artery with glue via the balloon protection technique $(\mathbf{G})$, complete occlusion of the AVF and aneurysmal dilatation was achieved $(\mathbf{H})$, although the spinal pial arteries from the ASA were left intact.

meningeal arteries from the VA (Table 1). In more than half of the lesions (63\%), the AV shunts were also fed by spinal arteries from the ASA and/or LSA. Moreover, the angiographic characteristics associated with hemorrhagic presentations comprised the inclusion of the ASA as a feeder, exclusion of the ECA as a feeder, the presence of aneurysmal dilatation of the feeder, and the CCJ AVF Type 2 (RAVF; Table 4). Treatment outcomes differed among the angiographic types of lesions. To our knowledge, this is the largest case series in the literature on CCJ AVFs in which the complex angioarchitecture was evaluated in detail (Table 5).

\section{Clinical Presentation and Angiographic Types}

In our report, hemorrhagic presentations were the most common symptom (SAH in 63\%, intramedullary hemorrhage in 10\%). Other studies also reported that SAH was a common presenting symptom in CCJ AVF. ${ }^{15,16}$ In Table 5, we summarized published CCJ AVF case series with hemorrhagic presentation. Historically, an ascending drainage route into an intracranial vein and venous varices were considered the causes of SAH. ${ }^{1,4-6,15,16}$ In addition, some authors have mentioned that higher venous flow rates are associated with intracranial drainage and the formation of varices leading to SAH. ${ }^{5,6}$ In contrast, 


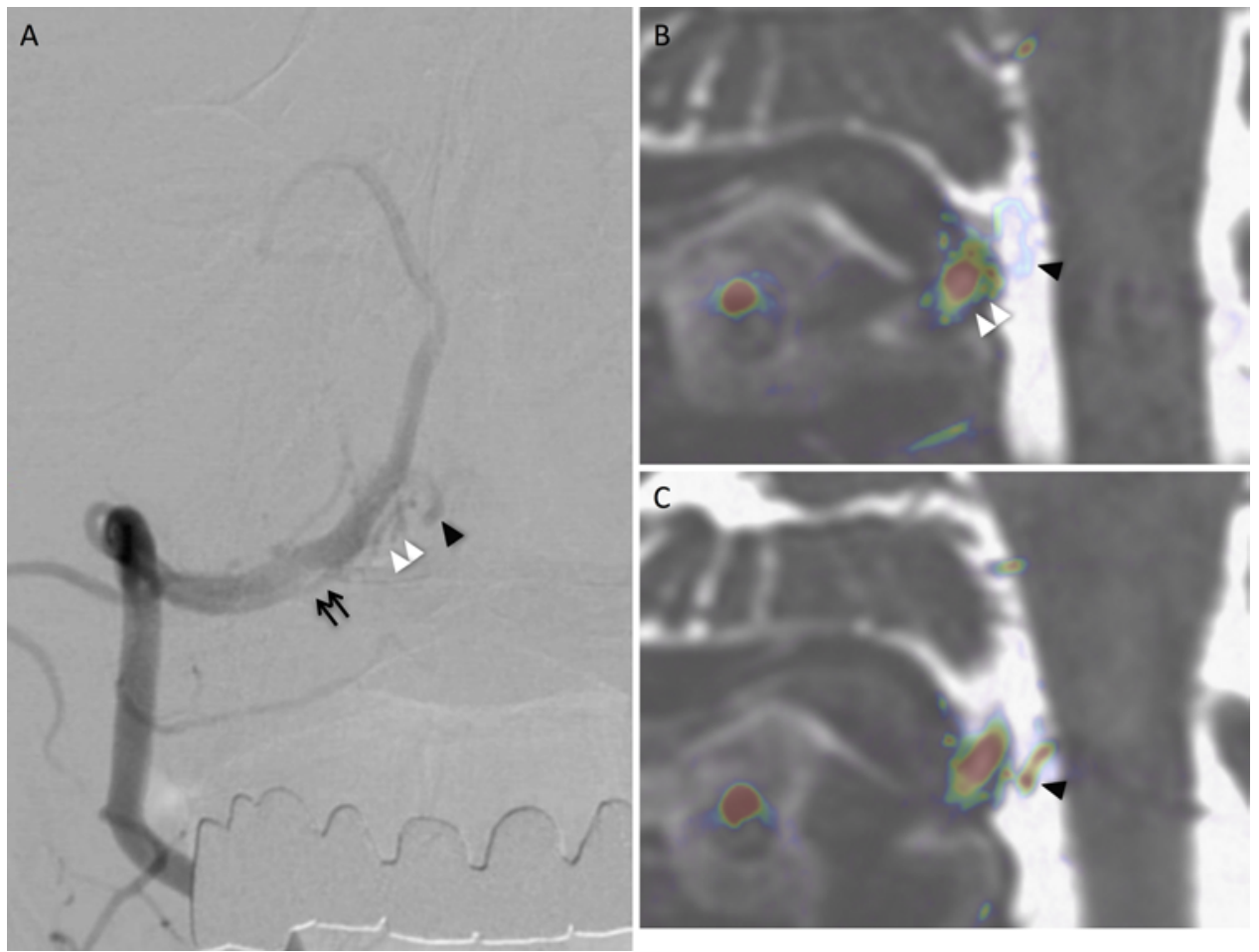

FIG. 4. Case 6. A CCJ EDAVF (Type 4) with no symptoms. Left VA angiogram (A), anteroposterior view, and 3D DSA/MRI fusion images $(B$ and $\mathbf{C})$ showing an EDAVF. The AVF is fed by the right $\mathrm{C}-1$ radiculomeningeal artery (double black arrows in $\mathrm{A}$ ). The feeder drains into the epidural venous plexus (double white arrowhead in $\mathrm{A}$ and $\mathrm{B}$ ) and the intradural vein (single black arrowhead in $\mathrm{A}-\mathrm{C}$ ). The $\mathrm{AV}$ shunt was presumed to be located in the epidural space.
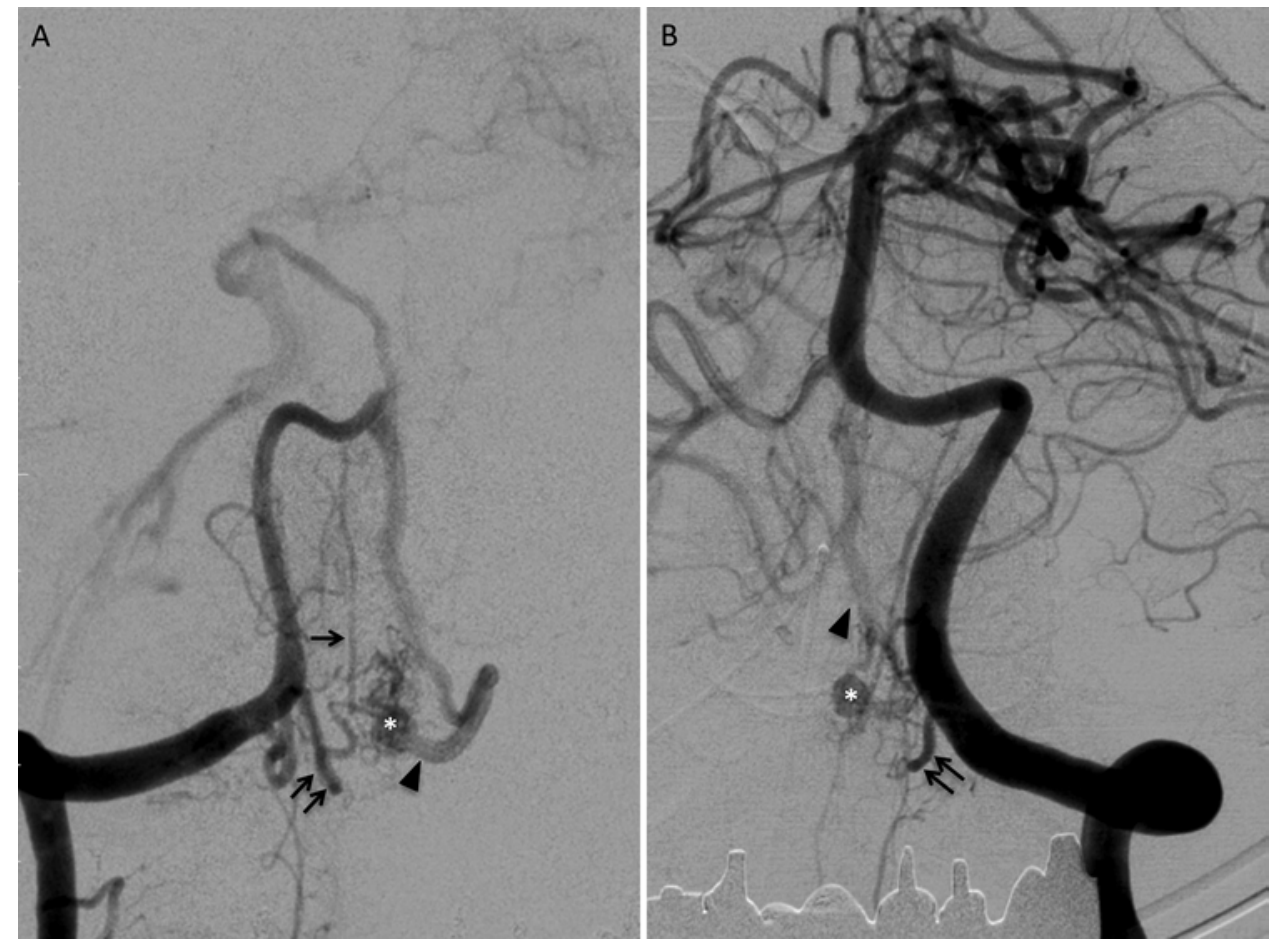

FIG. 5. Case 7. A CCJ PAVF (Type 5) presented with other symptoms. Right VA (A) and left VA (B) angiograms, anteroposterior views, showing a PAVF. The AVF is fed by the ASA (single arrow in A) and the lateral spinal arteries (double arrows in $A$ and $B$ ). Both feeders drain into the intradural vein (arrowheads in $A$ and $B$ ) with a varix (white asterisks in $A$ and $B$ ). The AV shunt was presumed to be located on the spinal cord. 
TABLE 1. Angioarchitecture by CCJ AVF type

\begin{tabular}{|c|c|c|c|c|c|c|}
\hline \multirow[b]{2}{*}{ Variable } & \multicolumn{6}{|c|}{ No. $(\%)$} \\
\hline & Type 1, DAVF & Type 2, RAVF & Type 3, EDAVF w/ Pial Feeders & Type 4, EDAVF & Type 5, PAVF & Total \\
\hline No. of lesions & 22 & 17 & 8 & 6 & 6 & 59 \\
\hline \multicolumn{7}{|l|}{ Lesion information } \\
\hline C-1 lesion & $22(100)$ & $13(76)$ & $2(25)$ & $2(33)$ & $3(50)$ & $42(71)$ \\
\hline C-2 lesion & 0 & $4(24)$ & $6(75)$ & $4(67)$ & $3(50)$ & $17(29)$ \\
\hline Rt side & $15(68)$ & $9(53)$ & $1(12)$ & $3(50)$ & $1(17)$ & $29(50)$ \\
\hline \multicolumn{7}{|l|}{ Feeder information } \\
\hline RMA & 100 & 100 & 100 & 100 & 83 & 98 \\
\hline ASA & 0 & $17(100)$ & $8(100)$ & 0 & $5(83)$ & $30(51)$ \\
\hline LSA & $5(23)$ & $5(29)$ & $3(38)$ & $1(17)$ & $2(33)$ & $16(27)$ \\
\hline ASA \&/or LSA & $5(23)$ & $17(100)$ & $8(100)$ & $1(17)$ & $6(100)$ & $37(63)$ \\
\hline ECA & $4(18)$ & $1(6)$ & 0 & $1(17)$ & $1(17)$ & $7(12)$ \\
\hline Aneurysmal dilatation & 0 & $10(59)$ & $8(100)$ & 0 & $1(17)$ & $19(32)$ \\
\hline \multicolumn{7}{|l|}{ Drainer information } \\
\hline Intradural drainage & $22(100)$ & $16(94)$ & $1(12)$ & $3(50)$ & $6(100)$ & $48(81)$ \\
\hline Ascending intradural drainage & $20(91)$ & $16(94)$ & 0 & $3(50)$ & $4(67)$ & $43(73)$ \\
\hline Epidural drainage & $1(4)$ & $6(35)$ & $8(100)$ & $6(100)$ & $1(17)$ & $22(37)$ \\
\hline Varix & $4(18)$ & $1(6)$ & 0 & 0 & $5(83)$ & $10(17)$ \\
\hline
\end{tabular}

$\mathrm{RMA}=$ radiculomeningeal artery .

we found that inclusion of the ASA as the feeder and aneurysmal dilatations were the risk factors associated with hemorrhagic presentation. Therefore, the CCJ AVF types associated with the ASA as a feeder, such as RAVF or EDAVF with pial feeders, developed SAH more frequently. Similarly, Sato et al. reported 9 cases of CCJ AVF in which 8 patients exhibited an arterial aneurysm on the distal side of the feeding arteries to the PAVF, and in each case, the aneurysm was intraoperatively confirmed as the bleeding point. ${ }^{13}$

\section{Radiculomeningeal and Pial Feeders of CCJ AVFs}

Much controversy exists regarding the diagnosis of CCJ AVFs because of the complexity of the angioarchitecture of these lesions. In reports published in the 1990s, CCJ AVFs were described as DAVFs fed by the radiculomeningeal artery. ${ }^{6,10}$ However, recent advances in angiography allow for the detection of fine vessels, and the spinal arteries have also been found to be involved in CCJ AVFs. In recent reports, the angiographic findings of CCJ AVFs were interpreted as the coincidental development of DAVF and PAVF. $5,11,13$

In the present study, we found that more than half of the CCJ AVFs (35 [59\%] of 59 lesions) were fed by both the radiculomeningeal arteries from the VA and the spinal pial arteries from the ASA and/or LSA. Moreover, 10 lesions with these 2 kinds of feeders were imaged with superselective angiography, and these feeders drained into the same vein. Furthermore, at least 6 patients underwent occlusion of the vein only (that is, spinal pial feeders were left intact), but the procedure resulted in complete occlusion of the $\mathrm{AVF}$ and aneurysmal dilatation of the pial feeder. We concluded that these AVFs are direct AV shunts that link both radiculomeningeal and pial feeders with the intradural or epidural vein at the same fistulous point. Considering the anatomical characteristics, we referred to these AVFs as RAVF or EDAVF with pial feeders (Fig. 6). However, because most of the patients with RAVF or EDAVF with pial feeders did not undergo superselective angiography of each vessel, it is not clear whether both vessels are true feeders of the AVF, or if one is the vessel feeding the AVF and the other is the vessel feeding the normal dura mater or the spinal cord. Furthermore, because of a lack of prior angiograms, it is not clear whether the association between the radiculomeningeal and pial feeders is causal or simply coincidental.

\section{Proposing the Concept "Radiculomeningeal AVF"}

In the present study, the locations of the AV shunts were presumed to be based on the change in the caliber between the feeding arteries and draining veins. There were some cases with angioarchitecture and shunt location typical for each type, which can be diagnosed easily; however, in most cases in this study, there were disagreements among the panel members in determining the complex angioarchitecture of CCJ AVFs. It was difficult to precisely localize the AV shunts in most patients because of the complicated arrangements of the fine feeding arteries and draining veins, the limited number of patients with superselective angiography of these feeders, and the lack of information about the neural structures.

Therefore, we propose that the CCJ AVFs should collectively be referred to as "radiculomeningeal AVFs." Radiculomeningeal AVFs have angiographic and clinical characteristics as follows. They have 5 types of angioarchitecture including DAVF, RAVF, EDAVF with pial feeders, EDAVF, and PAVF, and they develop along the C-1 or C-2 nerve roots. They commonly develop on the 
A
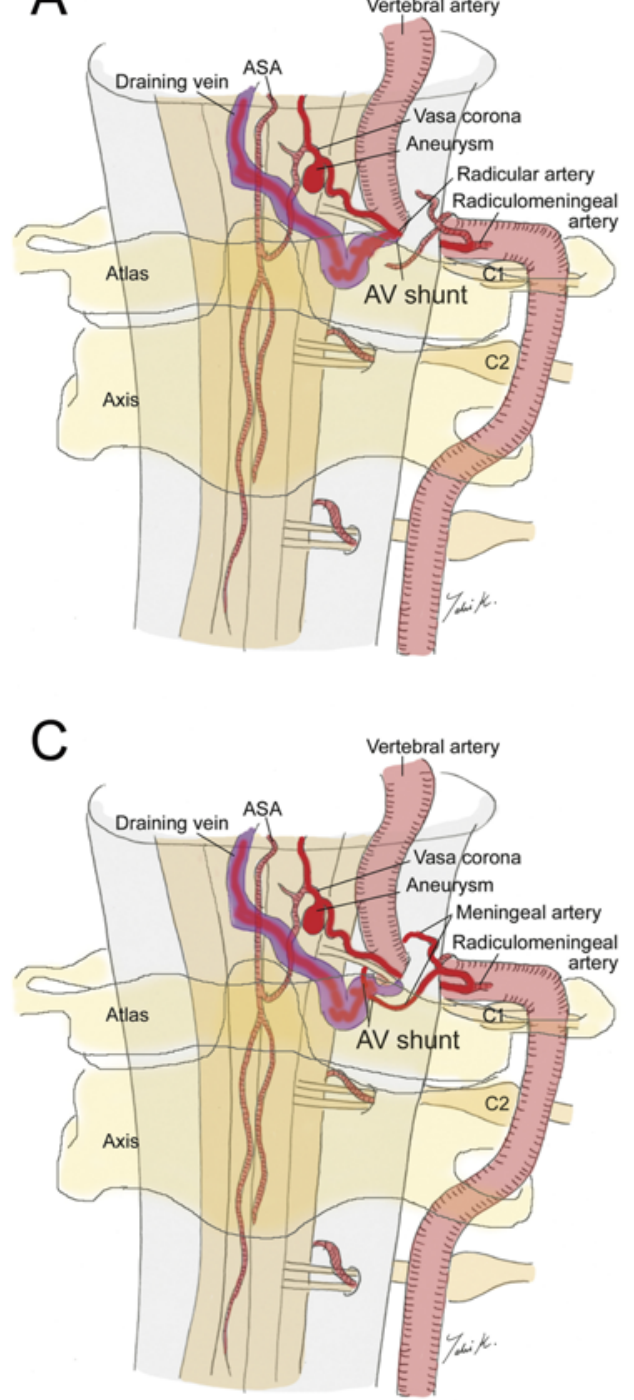
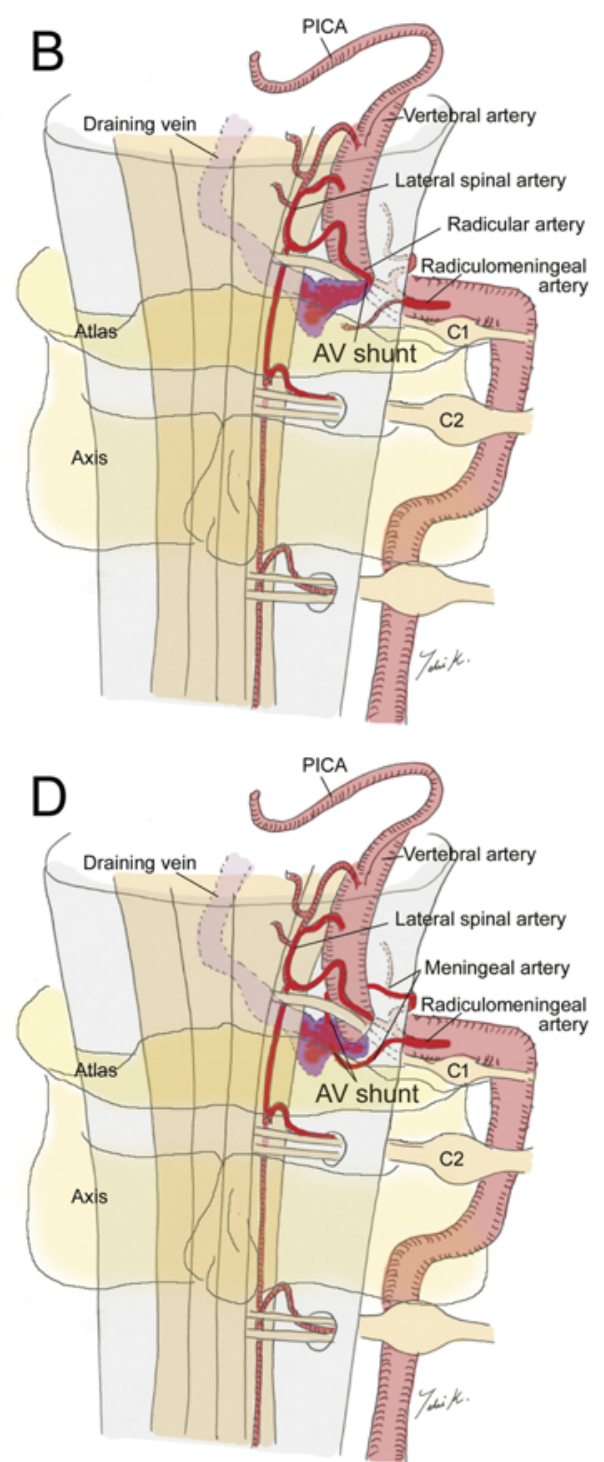

FIG. 6. Schematic illustrations of CCJ RAVF (Type 2). Angiographic interpretations, anteroposterior (A and C) and posteroanterior $(B$ and $D)$ views, of an RAVF ( $A$ and $B$ ) and a DAVF with pial feeders ( $C$ and $D)$. The AVF is fed by the radiculomeningeal artery that drains into the intradural vein through an AV shunt on the spinal nerve ( $A$ and $B$ ), where the pial branch of the ASA joins the same AV shunt and drains in a retrograde direction into the same intradural vein. Alternatively, the AVF is fed by the radiculomeningeal artery that drains into the intradural vein through an AV shunt on the dura mater ( $C$ and $D)$, where the pial branch of the ASA joins the same AV shunt and drains into the same intradural vein. The LSA can join the same intradural vein. PICA = posterior inferior cerebellar artery. Copyright Keisuke Takai. Published with permission.

TABLE 2. Clinical characteristics according to CCJ AVF type

\begin{tabular}{lcccccc}
\hline \multicolumn{1}{c}{ Variable } & Type 1, DAVF & Type 2, RAVF & Type 3, EDAVF w/ Pial Feeders & Type 4, EDAVF & Type 5, PAVF $^{\text {Overall* or Total }}$ \\
\hline No. of lesions & 22 & 17 & 8 & 6 & 6 & 59 \\
\hline Male sex (no. [\%]) & $18(82)$ & $12(71)$ & $5(62)$ & $6(100)$ & $4(67)$ & $45(76)$ \\
\hline Median age in yrs & 69 & 63 & 68 & 62 & 66 & $65^{*}$ \\
\hline Symptoms (no. [\%]) & & & & & \\
\hline SAH & $11(50)$ & $16(94)$ & $5(62)$ & 0 & $3(50)$ & $2(33)$ \\
\hline Venous congestion & $6(27)$ & 0 & $2(25)$ & 0 & $1(17)$ & $7(12)$ \\
\hline Intramedullary hemorrhage & $1(4)$ & 0 & $1(12)$ & $1(17)$ & $2(33)$ & $6(10)$ \\
\hline Other symptoms & $1(4)$ & 0 & 0 & 0 & $1(17)$ & $3(5)$ \\
\hline Asymptomatic & $3(14)$ & $1(6)$ & $2(33)$ & 0 & $6(10)$ \\
\hline
\end{tabular}

\footnotetext{
* Median value overall.
} 
TABLE 3. Treatment modality and outcome by CCJ AVF type

\begin{tabular}{|c|c|c|c|c|c|c|}
\hline Variable & Type 1, DAVF & Type 2, RAVF & Type 3, EDAVF w/ Pial Feeders & Type 4, EDAVF & Type 5, PAVF & Overall* or Total $^{*}$ \\
\hline No. of lesions & 22 & 17 & 8 & 6 & 6 & 59 \\
\hline \multicolumn{7}{|l|}{ Treatment (no. [\%]) } \\
\hline Direct surgery only & $15(68)$ & $6(35)$ & $2(25)$ & $3(50)$ & $2(33)$ & $28(47)$ \\
\hline IVR only & $3(14)$ & $4(24)$ & $4(50)$ & 0 & $4(67)$ & $15(25)$ \\
\hline Surgery + IVR & $2(9)$ & $5(29)$ & 0 & $1(17)$ & 0 & $8(14)$ \\
\hline Conservative & $2(9)$ & $2(12)$ & $2(25)$ & $2(33)$ & 0 & $8(14)$ \\
\hline \multicolumn{7}{|c|}{ Embolic material (no. [\%]) } \\
\hline Glue & $3(14)$ & $6(35)$ & $4(50)$ & 0 & $4(67)$ & $17(29)$ \\
\hline Coil & $3(14)$ & $3(18)$ & $1(12)$ & $1(17)$ & 0 & $8(14)$ \\
\hline \multicolumn{7}{|l|}{ Median mRS (IQR) } \\
\hline Preop & $3(1-3)$ & $1.5(1-3)$ & $3(1-5)$ & $2(0-4)$ & $5(3.5-5)$ & $3(1-4)^{*}$ \\
\hline Postop & $2(0.5-3)$ & $0.5(0-2)$ & $3(1-5)$ & $2(0-4)$ & $4(0.5-5)$ & $1(0-3)^{*}$ \\
\hline
\end{tabular}

IVR = interventional radiology.

* Overall value.

unilateral side at the C-1 or C-2 level, but sometimes they develop on bilateral sides or at multiple segmental levels. They are fed by the radiculomeningeal arteries from the VA, spinal pial arteries from the ASA and/or LSA, and the meningeal arteries from the ECA. The AV shunts were presumed to be located on the spinal cord, on the spinal nerves, and/or on the inner or outer surface of the dura mater (Fig. 7). They drain into intradural veins and/or epidural veins.

Radiculomeningeal AVFs commonly develop in men in their 60s. They are frequently associated with hemor-

TABLE 4. Risk factors associated with hemorrhagic presentation

\begin{tabular}{lccc}
\hline \multicolumn{1}{c}{ Variable } & RR & $95 \% \mathrm{Cl}$ & $\mathrm{p} \mathrm{Value}$ \\
\hline Patient information & & & \\
\hline Age $\geq 60$ yrs & 0.92 & $0.64-1.30$ & 0.7396 \\
\hline Sex: female & 1.24 & $0.93-1.66$ & 0.3102 \\
\hline Laterality: left & 1.27 & $0.93-1.74$ & 0.153 \\
\hline Feeder information & & & \\
\hline ASA & 1.40 & $1.02-1.92$ & 0.0439 \\
\hline LSA & 1.04 & $0.74-1.46$ & 1 \\
\hline ECA & 0.18 & $0.03-1.09$ & 0.001 \\
\hline Aneurysmal dilatation & 1.64 & $1.28-2.10$ & 0.0012 \\
\hline Drainer information & & & \\
\hline Intradural drainage & 0.87 & $0.62-1.21$ & 0.7099 \\
\hline Ascending intradural drainage & 0.93 & $0.67-1.29$ & 0.6932 \\
\hline Epidural drainage & 1.33 & $1.00-1.78$ & 0.1285 \\
\hline Varix & 1.30 & $0.98-1.71$ & 0.2592 \\
\hline CCJ AVF type & & & \\
\hline Type 1 (DAVF) as control & 1 & - & - \\
\hline Type 2 (RAVF) & 1.73 & $1.16-2.57$ & 0.0106 \\
\hline Type 3 (EDAVF w/ pial feeders) & 1.60 & $1.01-2.55$ & 0.1987 \\
\hline Type 4 (EDAVF) & 1.22 & $0.62-2.42$ & 0.673 \\
\hline Type 5 (PAVF) & 1.22 & $0.61-2.42$ & 0.673 \\
\hline
\end{tabular}

rhagic presentations including $\mathrm{SAH}$ and intramedullary hemorrhage, especially in patients with spinal feeders from the ASA (RR 1.40), without meningeal feeders from the ECA (RR 0.18), with aneurysmal dilatation of the pial feeders (RR 1.64), or with the CCJ AVF Type 2, RAVF (RR 1.73).

\section{Study Limitations}

The present study has some methodological limitations. First, it is difficult to precisely localize the AV shunts of CCJ AVFs based on conventional angiography alone. For a preoperative diagnosis, multimodal fusion images from 3D rotational angiography and MRI or CT myelography may be useful because these images provide simultaneous stereoscopic visualization of the vascular system and neural structures. ${ }^{14}$ For intraoperative diagnosis, test occlusion of the drainage vein before occlusion of the feeding arteries may be useful to assess whether both radiculomeningeal and spinal feeding arteries connect to the same vein at the same fistulous point or not. Second, this study was conducted using a retrospective approach. Third, we could
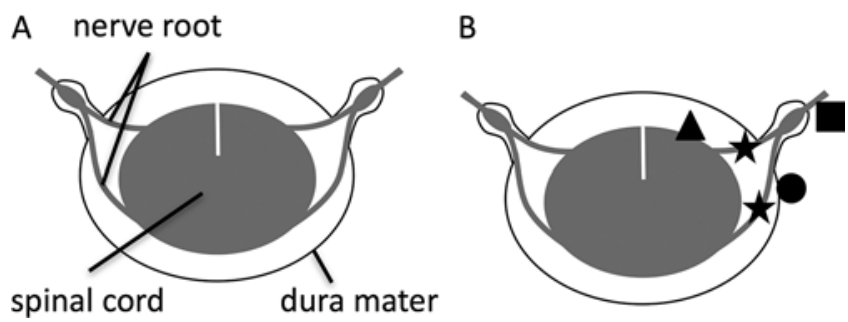

FIG. 7. Schematic illustrations showing possible AV shunt locations of CCJ AVFs. A: Transaxial plane of spinal anatomical structures. B: Possible AV shunt locations of CCJ AVFs. The AV shunt develops along the $\mathrm{C}-1$ or $\mathrm{C}-2$ nerve roots and can be located on the spinal cord, on the spinal nerves, and/or on the inner or outer surface of the dura mater. This includes Types 1-5 CCJ AVFs. Circle indicates the shunt point of the DAVF. Stars indicate the shunt points of the RAVF. Square indicates the shunt point of the EDAVF. Triangle indicates the shunt point of the PAVF. 
TABLE 5. Summary of reported CCJ AVF case series with hemorrhagic presentation

\begin{tabular}{lccl}
\hline \multicolumn{1}{c}{ Authors \& Year } & No. of Cases & Hemorrhage Cases & \multicolumn{1}{c}{ Cause of Hemorrhage } \\
\hline Kinouchi et al., 1998 & 10 & $70 \%$ & Venous drainage mainly in the superior direction, fast flow, varix \\
\hline Aviv et al., 2004 & 2 & $100 \%$ & Intracranial or superior drainage, varix \\
\hline Kai et al., 2005 & 6 & $100 \%$ & Ascending venous drainage \\
\hline Kim et al., 2010 & 12 & $42 \%$ & Rostral intracranial venous drainage \\
\hline Sato et al., 2013 & 9 & $100 \%$ & Feeder aneurysm \\
\hline Onda et al., 2014 & 3 & $66 \%$ & Ascending venous drainage, aneurysm of a feeder from the ASA \\
\hline Wang et al., 2015 & $127^{*}$ & $43 \%$ & Ascending venous drainage \\
\hline Zhao et al., 2015 & $56^{*}$ & $38 \%$ & Intracranial venous drainage, varix \\
\hline Present study & 59 & $73 \%$ & Inclusion of ASA as a feeder, aneurysmal dilatation on the feeder, CCJ AVF Type 2 (RAVF) \\
\hline
\end{tabular}

* Review.

not confirm the operative findings in all lesions. Fourth, although the present study was a relatively large series, the number of cases for each type is small. In the future, a prospective nationwide study with a large number of patients using advanced imaging techniques and detailed operative findings should be performed.

\section{Conclusions}

We propose a new classification system based on angiographic characteristics because the angioarchitecture of CCJ AVFs is more complex than previously recognized. These AVFs are commonly associated with hemorrhage and are frequently fed by both radiculomeningeal and spinal pial arteries that drain into intradural or epidural veins. The AV shunt develops along the C-1 or C-2 nerve roots and can be located on the spinal cord, on the spinal nerves, and/or on the inner or outer surface of the dura mater.

\section{Acknowledgments}

We express our heartfelt thanks to the collaborating doctors who devoted their time to this investigation: Akira Ishii and Ryota Ishibashi, Department of Neurosurgery, Kokura Memorial Hospital, Fukuoka; Hirofumi Nakatomi, Department of Neurosurgery, Graduate School of Medicine, The University of Tokyo, Tokyo; Ichiro Nakahara and Sadayoshi Watanabe, Department of Comprehensive Strokology, Fujita Health University of Medicine, Aichi; Makoto Sakamoto, Division of Neurosurgery, Department of Brain and Neurosciences, Faculty of Medicine, Tottori University, Tottori; Masaki Komiyama, Department of Neuro-Intervention, Osaka City General Hospital, Osaka; Masaru Hirohata and Yukihiko Nakamura, Department of Neurosurgery, Kurume University School of Medicine, Fukuoka; Masayuki Sato, Department of Endovascular Neurosurgery, Tranomon Hospital, Tokyo; Michiyasu Suzuki, Hideyuki Ishihara, and Hisaharu Goto, Department of Neurosurgery and Clinical Neuroscience, Yamaguchi University School of Medicine, Yamaguchi; Naoki Akioka and Naoya Kuwayama, Department of Neurosurgery, Graduate School of Medicine and Pharmacological Sciences, University of Toyama, Toyama; Osamu Masuo, Department of Neurological Surgery, Wakayama Medical University, Wakayama; Shigeru Miyachi, Department of Neurosurgery, Osaka Medical College, Osaka; Shuichi Tanoue, Department of Radiology, Oita University Faculty of Medicine, Oita; Tomoaki Terada, Department of Neurosurgery, Showa University Fujigaoka Hospital, Kanagawa; Tsuyoshi Asano, Department of Neurosurgery, Asahikawa Red Cross Hospital, Hokkaido; Yasushi Matsumoto, Department of Neuroendovascular
Therapy, Kohnan Hopital, Miyagi; Wataro Tsuruta, Department of Neurosurgery, Faculty of Medicine, University of Tsukuba, Tsukuba, Japan.

\section{References}

1. Aviv RI, Shad A, Tomlinson G, Niemann D, Teddy PJ, Molyneux AJ, et al: Cervical dural arteriovenous fistulae manifesting as subarachnoid hemorrhage: report of two cases and literature review. AJNR Am J Neuroradiol 25:854-858, 2004

2. Endo T, Shimizu H, Sato K, Niizuma K, Kondo R, Matsumoto Y, et al: Cervical perimedullary arteriovenous shunts: a study of 22 consecutive cases with a focus on angioarchitecture and surgical approaches. Neurosurgery 75:238-249, 2014

3. Hiramatsu M, Sugiu K, Hishikawa T, Haruma J, Tokunaga K, Date I, et al: Epidemiology of dural arteriovenous fistula in Japan: Analysis of Japanese Registry of Neuroendovascular Therapy (JR-NET2). Neurol Med Chir (Tokyo) 54:63-71, 2014

4. Kai Y, Hamada J, Morioka M, Yano S, Mizuno T, Kuratsu J: Arteriovenous fistulas at the cervicomedullary junction presenting with subarachnoid hemorrhage: six case reports with special reference to the angiographic pattern of venous drainage. AJNR Am J Neuroradiol 26:1949-1954, 2005

5. Kim DJ, Willinsky R, Geibprasert S, Krings T, Wallace C, Gentili F, et al: Angiographic characteristics and treatment of cervical spinal dural arteriovenous shunts. AJNR Am J Neuroradiol 31:1512-1515, 2010

6. Kinouchi H, Mizoi K, Takahashi A, Nagamine Y, Koshu K, Yoshimoto T: Dural arteriovenous shunts at the craniocervical junction. J Neurosurg 89:755-761, 1998

7. Klopper HB, Surdell DL, Thorell WE: Type I spinal dural arteriovenous fistulas: historical review and illustrative case. Neurosurg Focus 26(1):E3, 2009

8. Krings T, Geibprasert S: Spinal dural arteriovenous fistulas. AJNR Am J Neuroradiol 30:639-648, 2009

9. Kuwayama N, Endo S, Kubo M, Sakai N: [Present status in the treatment of dural arteriovenous fistulas in Japan.] Jpn J Neurosurg (Tokyo) 20:12-19, 2011 (Jpn)

10. Niwa J, Matsumura S, Maeda Y, Ohoyama H: Transcondylar approach for dural arteriovenous fistulas of the cervicomedullary junction. Surg Neurol 48:627-631, 1997

11. Onda K, Yoshida Y, Watanabe K, Arai H, Okada H, Terada T: High cervical arteriovenous fistulas fed by dural and spinal arteries and draining into a single medullary vein: report of 3 cases. J Neurosurg Spine 20:256-264, 2014

12. Reinges MH, Thron A, Mull M, Huffmann BC, Gilsbach JM: Dural arteriovenous fistulae at the foramen magnum. J Neurol 248:197-203, 2001 
13. Sato K, Endo T, Niizuma K, Fujimura M, Inoue T, Shimizu $\mathrm{H}$, et al: Concurrent dural and perimedullary arteriovenous fistulas at the craniocervical junction: case series with special reference to angioarchitecture. J Neurosurg 118:451-459, 2013

14. Takai K, Kin T, Oyama H, Iijima A, Shojima M, Nishido H, et al: The use of 3D computer graphics in the diagnosis and treatment of spinal vascular malformations. J Neurosurg Spine 15:654-659, 2011

15. Wang JY, Molenda J, Bydon A, Colby GP, Coon AL, Tamargo RJ, et al: Natural history and treatment of craniocervical junction dural arteriovenous fistulas. J Clin Neurosci 22:1701-1707, 2015

16. Zhao J, Xu F, Ren J, Manjila S, Bambakidis NC: Dural arteriovenous fistulas at the craniocervical junction: a systematic review. J Neurointerv Surg 8:684-653, 2016

\section{Disclosures}

The authors report no conflict of interest concerning the materials or methods used in this study or the findings specified in this paper.

\section{Author Contributions}

Conception and design: Sugiu, Kiyosue, Matsumaru. Acquisition of data: Hiramatsu, Sugiu, Matsumaru. Analysis and interpretation of data: all authors. Drafting the article: Hiramatsu, Sugiu. Critically revising the article: all authors. Reviewed submitted version of manuscript: all authors. Approved the final version of the manuscript on behalf of all authors: Hiramatsu. Statistical analysis: Hiramatsu. Study supervision: Sugiu, Matsumaru.

\section{Supplemental Information}

\section{Current Affiliations}

Dr. Matsumaru: Division for Stroke Prevention and Treatment, Department of Neurosurgery, Faculty of Medicine, University of Tsukuba, Ibaraki, Japan.

\section{Correspondence}

Masafumi Hiramatsu, Department of Neurological Surgery, Okayama University Graduate School of Medicine, Dentistry and Pharmaceutical Sciences, 2-5-1 Shikata-cho, Kita-ku, Okayama 700-8558, Japan. email: mhiramatsu@okayama-u.ac.jp. 